\title{
The Radiative Strength Function Using the Neutron-Capture Reaction on 151,153Eu
}

U. Agvaanluvsan, A. Alpizar-Vicente, J. A. Becker, F. Becvar, T. A. Bredeweg, R. Clement, E. Esch, C. M. Folden, III, R. Hatarik, R. C. Haight, D. C. Hoffman, M. Krticka, R. A. Macri, G. E. Mitchell, H.

Nitsche, J. M. O'Donnell, W. Parker, R. Reifarth, R. S. Rundberg, J. M. Schwantes, S. A. Sheets, J. L. Ullmann, D. J. Vieira, J. B. Wilhelmy, P. Wilk, J. M. Wouters, C. Y. Wu

\section{October 6, 2005}

12th Conference on Capture Gamma-ray Spectroscopy and Related Topics, Notre-Dame South Bend, IN, United States September 4, 2005 through September 9, 2005 
This document was prepared as an account of work sponsored by an agency of the United States Government. Neither the United States Government nor the University of California nor any of their employees, makes any warranty, express or implied, or assumes any legal liability or responsibility for the accuracy, completeness, or usefulness of any information, apparatus, product, or process disclosed, or represents that its use would not infringe privately owned rights. Reference herein to any specific commercial product, process, or service by trade name, trademark, manufacturer, or otherwise, does not necessarily constitute or imply its endorsement, recommendation, or favoring by the United States Government or the University of California. The views and opinions of authors expressed herein do not necessarily state or reflect those of the United States Government or the University of California, and shall not be used for advertising or product endorsement purposes. 


\title{
The Radiative Strength Function Using the Neutron-Capture Reaction on ${ }^{151,153} \mathrm{Eu}$
}

\author{
U. Agvaanluvsan, ${ }^{*}$ A. Alpizar-Vicente, ${ }^{\dagger}$ J.A. Becker, ${ }^{*}$ F. Bečvár, \\ T.A. Bredeweg, ${ }^{* *}$ R. Clement, ${ }^{*}$ E. Esch, ${ }^{* *}$ C.M. Folden, III" R. Hatarik, ${ }^{\dagger}$ \\ R.C. Haight, ${ }^{* *}$ D.C. Hoffman, ${ }^{\natural}$ M. Krtička, ${ }^{\ddagger}$ R.A. Macri, \\ G.E. Mitchell, ${ }^{\S}$ H. Nitsche, ${ }^{\natural}$ J.M. O'Donnell, ${ }^{* *}$ W. Parker, ${ }^{*}$ R. Reifarth, ${ }^{*}$ \\ R.S. Rundberg, ${ }^{* *}$ J.M. Schwantes, ${ }^{* *}$ S.A. Sheets, ${ }^{* *}$ J.L. Ullmann, ${ }^{* *}$ \\ D.J. Vieira, ${ }^{* *}$ J.B. Wilhelmy, ${ }^{* *}$ P. Wilk, ${ }^{*}$ J.M. Wouters, ${ }^{* *}$ C.Y. Wu \\ *Lawrence Livermore National Laboratory, Livermore, CA 94551 \\ ${ }^{\dagger}$ Colorado School of Mines, Golden, CO 80401 \\ **Los Alamos National Laboratory, Los Alamos, NM 87545 \\ ${ }^{\ddagger}$ Charles University in Prague, CZ-180 00 Prague 8, Czech Republic \\ ${ }^{\S}$ North Carolina State University, Raleigh, NC 27695 and \\ Triangle University Nuclear Laboratory, Durham, NC 27708 \\ "University of California Berkeley and Lawrence Berkeley National Laboratory, Berkeley, CA 94720
}

\begin{abstract}
Radiative strength functions in ${ }^{152,154} \mathrm{Eu}$ nuclei for $\gamma$-ray energies below 6 $\mathrm{MeV}$ have been investigated. Neutron capture for incident neutron energies $<1 \mathrm{eV}$ up to $100 \mathrm{keV}$ has been measured for ${ }^{151,153}$ Eu targets. Properties of resonances in these two nuclei are examined. The measurements are compared to simulation of cascades performed with various models for the radiative strength function. Comparison between experimental data and simulation suggests an existence of the low-energy resonance in these two nuclei.
\end{abstract}

Keywords: neutron capture, radiative strength function, resonances.

PACS: 24.30.-v, 24.30.Gz, 24.30.Gd

\section{INTRODUCTION}

Nuclear radiative strength functions are known best around the maximum of the giant electric dipole resonance, $10-20 \mathrm{MeV} \gamma$-ray energy. Extrapolation of the radiative strength functions from the giant dipole resonance region to lower $\gamma$-ray energy region is problematic at best. The radiative strength function, also known as the $\gamma$-ray or photon strength function is defined [1] as

$$
f_{X L}\left(E_{\gamma}\right)=\frac{\left\langle\Gamma_{\gamma i f}\right\rangle}{E_{\gamma}^{2 L+1} D_{i}},
$$

where $\left\langle\Gamma_{\text {rif }}\right\rangle$ is the average partial radiative width for transition from an initial state $i$ to a final state $f, E_{\gamma}$ is the energy of transition, and $D_{i}$ is the level spacing of the initial states. Most experimental data for the RSF is from the study of photoabsorption cross sections [2,3]. Other methods involving radiative neutron capture such as the spectrum fitting method [4] and the two-step cascade method $[5,6]$ provide additional 
information about the RSF for high-energy transitions. The investigation of primary $\gamma$ rays of different multipolarities [7, 8] and the sequential extraction method [9] are also used for obtaining experimental information for the RSF.

One of the most commonly used theoretical models is the Lorentzian function for the Giant Electric Dipole Resonance (GEDR). For deformed nuclei, experimental data can be fit as a superposition of two Lorentzians. The Giant Magnetic Dipole (or Spin-flip) Resonance (GMDR) is also described by the Lorentzian [7, 10]. Although the Lorentzian model describes data at higher transition energies, it does not adequately describe the data for low energy $\gamma$-rays [11]. To explain the non-zero limit of the RSF for $E_{\gamma} \rightarrow 0$, models based on the Fermi liquid theory were developed $[12,13]$ that give an energy and temperature dependent damping width. The strength function model developed by Kadmenskiĭ, Markushev, and Furman (KMF) is given by

$$
f_{E 1}\left(E_{\gamma}\right)=\frac{1}{3 \pi^{2} \hbar^{2} c^{2}} \frac{0.7 \sigma_{E 1} \Gamma_{E 1}^{2}\left(E_{\gamma}^{2}+4 \pi^{2} T^{2}\right)}{E_{E 1}\left(E_{\gamma}^{2}-E_{E 1}^{2}\right)^{2}},
$$

where $\Gamma_{E 1}$ is energy and temperature dependent. In addition to the GEDR and GMDR, the low energy $\left(E_{\gamma} \sim 3 \mathrm{MeV}\right.$ ) mode with the Lorentzian shape is considered for the total RSF.

The experimental indication of the low energy mode has been observed in several types of measurements. In the so-called Oslo method, the level density and radiative strength function are obtained simultaneously [14]. In deformed rare earth nuclei studied with the Oslo-method, a resonance near $E_{\gamma} \sim 3 \mathrm{MeV}$ is observed and is identified as a pygmy resonance $[15,16]$. In another set of experiments by a nuclear resonance fluorescence (NRF) method, a resonance mode at around the same energy, $E_{\gamma} \sim 3 \mathrm{MeV}$ is observed [17]. The multipolarity is unambiguously determined as an $\mathrm{M} 1$ and the resonance is called a scissors mode (SM) resonance. The A-dependences of the resonance energy from above two sets of experiments do not agree. In addition, the total strengths of the pygmy resonance observed in the Oslo-type experiments are larger than the SM strength observed by the NRF. Therefore, it is unclear whether the same physics phenomenon explains both effects fully. In the so called two-step cascade (TSC) method, de-excitation of nucleus following the thermal neutron capture is studied [18] where the SM resonance is also observed. However the strength is again greater than that of observed in the NRF experiments. From the TSC experiment it was found that a SM resonance in ${ }^{163}$ Dy is built on all excited levels [19]. DANCE data reveal similar phenomenon in the statistical $\gamma$-ray decay cascade of Eu nuclei.

\section{EXPERIMENTAL DESCRIPTION}

The experiment was performed using the DANCE array located at the flight path 14 at Lujan Center at the Los Alamos Neutron Science Center (LANSCE). The DANCE array is a $4 \pi \gamma$-ray calorimeter that consists of 160 barium fluoride crystals. The neutron beam with $E_{n}=10 \mathrm{meV}-100 \mathrm{keV}$ with the repetition rate $20 \mathrm{~Hz}$ was provided by the spallation neutron source at LANSCE. The flight path length is 20 
meter. The neutron energy is determined by the time-of-flight technique. The stable ${ }^{151,153} \mathrm{Eu}$ targets with thicknesses $0.836 \pm 0.040 \mathrm{mg} / \mathrm{cm}^{2}$ and $1.06 \pm 0.05 \mathrm{mg} / \mathrm{cm}^{2}$ and enrichment $96.83 \%$ and $98.76 \%$, respectively, were used. Both targets were mounted on a Be backing. The DANCE data acquisition system relies on waveform digitization. The description of the DAQ is given in reference [20]. The details of various background and methods of suppression are described in reference [21]. Event by event data analysis was performed offline. Taking advantage of the high granularity of the DANCE detector, events for each multiplicity can be separated as function of neutron energy. Various neutron energy regions and spectrum of events for each multiplicity can be selected as shown in Fig. 1 for the two resonances in ${ }^{152} \mathrm{Eu}$.

FIGURE 1. The first two resonances in the ${ }^{151} \mathrm{Eu}(\mathrm{n}, \gamma)$ reaction. Events with different $\gamma$-ray multiplicities can be separated for each resonance. The most dominant are multiplicities 3 and 4.

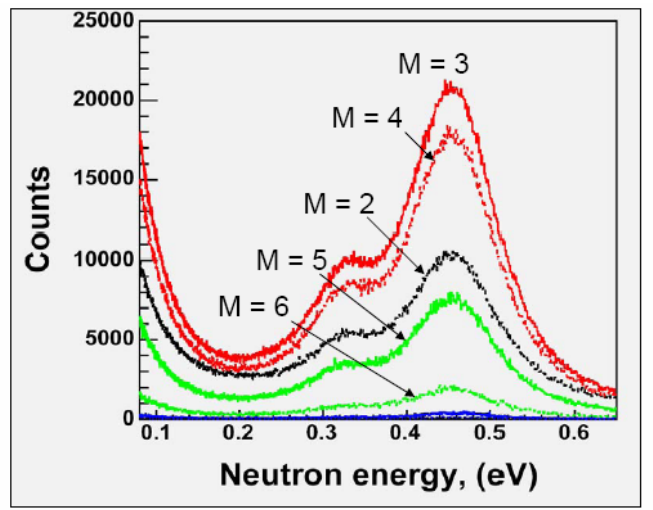

\section{SIMULATION AND MODELING}

Experimental data obtained from the DANCE detector can be compared with an outcome from simulations performed by adjacent DICEBOX/GEANT simulations. The Monte Carlo code DICEBOX [18] generates $\gamma$-ray cascades initiating at the neutron capturing state and terminating at the ground state following the rules of the extreme statistical model. The level system of the nucleus and the associated decay scheme are artificially generated according to adapted level-density and radiative strength function models. Each set of the generated level structure and the decay scheme are called a nuclear realization. The level structure below a critical energy $\left(\mathrm{E}_{\text {crit }} \sim 400 \mathrm{keV}\right.$ in odd-odd $\left.\mathrm{Eu}\right)$ is taken from a known level scheme [22] and kept fixed. Above $\mathrm{E}_{\text {crit }}$, the level density and decay scheme are assumed to follow statistical rules. Many nuclear realizations are simulated. Introducing the technique of precursors, as described in [18], the code DICEBOX offers the unique feature of simulation of the residual Porter-Thomas fluctuations. Another important feature of the code is the treatment of conversion electrons which is a considerable factor for the decay of Eu nuclei.

Cascades produced by the DICEBOX code in the list mode serve as an input for GEANT simulation of the detector response to these cascades. Various types of 
simulated spectra are produced. Simulation and data for sum-energy spectra and $\gamma$-ray spectra for events for various multiplicities with deposited sum-energy close to Qvalue of the $(n, \gamma)$ reaction are compared. Comparisons between data from the Eu experiments at DANCE and simulation are shown in Fig. 2. Multiplicity 2 (top graphs) and multiplicity 3 (bottom graphs) spectra are shown as a function of the $\gamma$-ray energy. Experimental data are shown in black histograms and simulation is shown in red. In the experimental multiplicity 2 spectrum cascades consisting of two-step transitions are selected, thus the spectrum is symmetric around the center as expected. The peak on the right hand side is slightly broader than the peak on the left due to the poorer detector resolution for higher energy $\gamma$-rays. The bump structure in the center of the multiplicity 2 spectrum, and near $2.5 \mathrm{MeV}$ in the multiplicity 3 spectrum is identified to be due to the existence of the low-energy mode resonance.

FIGURE 2. Comparison between data and simulation. The black histograms represent data from the neutron energy $E_{n}=0.24-0.65 \mathrm{eV}$ gated around the first two resonances in the ${ }^{151} \mathrm{Eu}(\mathrm{n}, \gamma)$ reaction. The red histograms represent simulations a) without postulating any resonance and 2) with postulating an M1 resonance.

a)

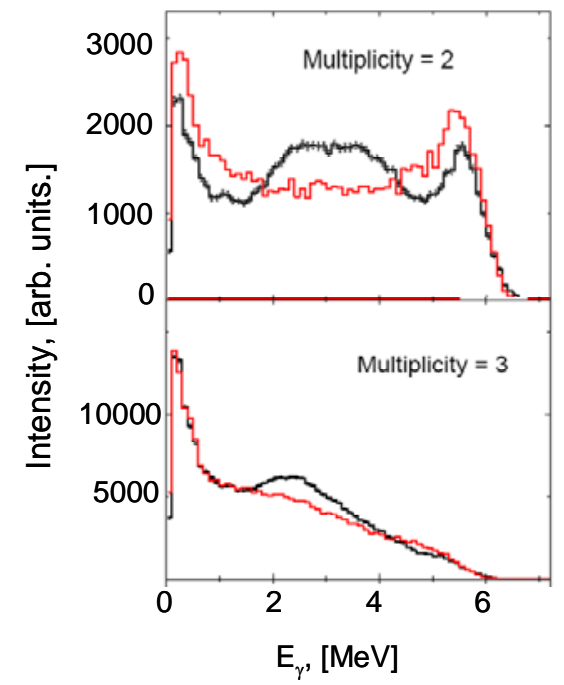

b)

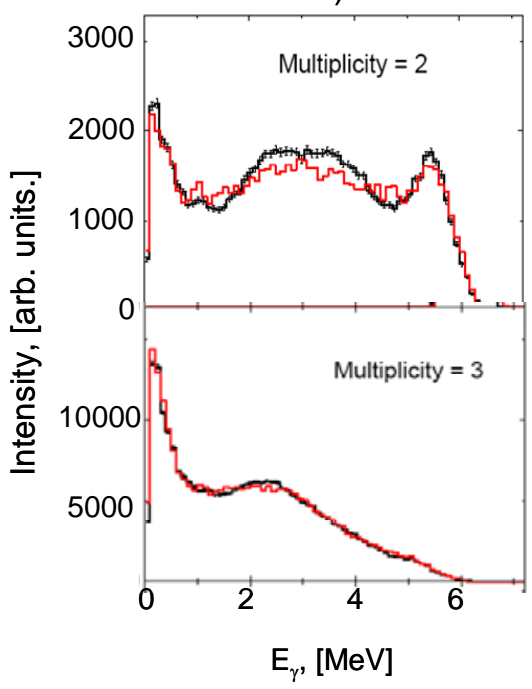

In both a) and b), the Back-Shifted Fermi gas model for the level density is employed. For the radiative strength function, a combination of the KMF (for lower $\mathrm{E}_{\gamma}$ ) and Lorentzian (for higher $\mathrm{E}_{\gamma}$ ) models for the E1 GEDR and the M1 spin-flip GMDR given by the Lorentzian are chosen. Without introducing any low energy resonance near $3 \mathrm{MeV}$, an agreement between data and simulation for all multiplicities is poor. As an example of poor fit, multiplicity 2 and 3 spectra are shown in the two graphs on the left in Fig. 2. Assuming an E1 resonance near $3 \mathrm{MeV}$ does not improve the fit. Assuming an M1 resonance improves the fit significantly. Variation of the parameters of the M1 resonance yields better fit at $\mathrm{E}_{\gamma}=2.6 \mathrm{MeV}$ with width $\Gamma_{\gamma}=1.6$, with a good agreement in the multiplicity 3 spectrum, an improved but not satisfactory agreement in the multiplicity 2 spectrum as shown in the right two graphs in Fig. 2. Similar resonance is also observed in the case of ${ }^{153} \mathrm{Eu}$. The complicated level structure of 
odd-odd compound systems ${ }^{152,154} \mathrm{Eu}$ requires further refinement in the consideration of effect of isomers and varying deformation as a function of excitation energy.

\section{CONCLUSION AND OUTLOOK}

The $4 \pi \gamma$-ray calorimeter DANCE is utilized for the study of statistical decay cascade following the neutron capture on stable Eu targets. The high granularity of the detector allows one to look closely at cascades with specified number of transitions. The decay cascade is simulated using the statistical code DICEBOX taking into account the detector response function with usage of the GEANT code. The outcome of the simulation is compared with the experimental spectra. A concentration of $\gamma$-ray strength near $\mathrm{E}_{\gamma} \sim 2.5 \mathrm{MeV}$ is identified at present as manifestation of the scissorsmode like mechanism in odd-odd Eu nuclei. Further developments in the analysis and simulation are underway.

Acknowledgement. Work supported in part by the U.S. DOE by the UC under contract Nos. W-7405-ENG-48 (LLNL) and W-7405-ENG-36 (LANL), and by the NNSA Academic Alliance through DOE Research Grant Nos. DE-FG03-03-NA00076 (NCSU) and DE-FG0303-NA00075 (UCB). Support by U.S. Department of Energy Grant No. DE-FG02-97ER41042 (NCSU/TUNL) is acknowledged.

\section{References:}

[1] J. M. Blatt and V. F. Weisskopf, Theoretical Nuclear Physics, Wiley, New York, (1952).

[2] B. L. Berman and S. C. Fultz, Rev. Mod. Phys. 47, 713 (1975).

[3] Samuel S. Dietrich and Barry L. Berman, At. Data Nucl. Data Tables 38, 199 (1988).

[4] E. D. Earle et al., Can. J. Phys. 52, 989 (1974).

[5] F. Bečvář, P. Cejnar, R. E. Chrien, and J. Kopecky, Phys. Rev. C 46, 1276 (1992).

[6] F. Bečvář, P. Cejnar, J. Honzátko, K. Konečný, I. Tomandl, and R. E. Chrien, Phys. Rev. C 52, 1278 (1995).

[7] J. Kopecky and R. E. Chrien, Nucl. Phys. A468, 285 (1987).

[8] W. V. Prestwich et al., Z. Phys. A315, 103 (1984).

[9] G. A. Bartholomew et al., Can. J. Phys. 48, 687 (1970).

[10] A. Richter, Prog. Part. Nucl. Phys. 34, 261 (1995).

[11] Y. P. Popov, Fiz. Elem. Chastits. At. Yadra 13, 1165 (1982), [Sov. J. Part. Nucl. 13, 483 (1982)].

[12] S. G. Kadmensiǔ, V. P. Markushev, and V. I. Furman, Yad. Fiz. 37, 277 (1983), [Sov. J. Nucl. Phys. 37, 165 (1983)].

[13] V. K. Sirotkin, Yad. Fiz. 43, 570 (1986), [Sov. J. Nucl. Phys. 43, 362 (1986)].

[14] M. Guttormsen et al., Phys. Scr. T32, 54 (1990).

[15] M. Guttormsen et al., Phys. Rev. C 68, 064306 (2003).

[16] U. Agvaanluvsan et al., Phys. Rev. C 70, 054611 (2004).

[17] A. Zilges et al., Nucl. Phys. A519, 848 (1990).

[18] F. Bečvář, Nucl. Instr. Methods A 417, 434 (1998).

[19] M. Krtička et al., Phys. Rev. Lett. 92, 172501 (2004).

[20] J. M. Wouters et al., to be published in the Proceedings of the $14^{\text {th }}$ IEEE-NPSS

Real-Time Conference, (2005).

[21] R. Reifarth et al., Nucl. Inst. Methods Phys. Res. A 531, 530 (2004).

[22] T. Von Egidy et al., Z. Phys. A 286, 241 (1978). 